\title{
Hemi-Thyroidectomy versus Total Thyroidectomy in Management of Papillary Thyroid Carcinoma
}

\author{
E.S.Radwan, A.A.Salem, A.M.Salama and M.M.Amin \\ general surgery, Dept., Faculty of Medicine, Benha Univ., Benha, Egypt \\ E-Mail: mohamedaminsalmawy@gmail.com
}

\begin{abstract}
Papillary thyroid cancer (PTC) is the most common form of thyroid cancer, accounting for nearly $70 \%$ of all thyroid tumours. In terms of thyroid cancer, differentiated thyroid carcinoma is the most prevalent kind. A good prognosis may be expected if the cancer has not spread to the lymph nodes or distant organs, however this is dependent on the kind of cancer and other prognostic markers such as age, gender, lymph node involvement, and distant metastasis. As many as $11 \%$ to $30 \%$ of patients with early stage I and II PTC relapse after surgical resection, according to certain studies. There are several variables to consider when determining how extensive a patient's surgery should be, including the tumor's size, how far it has spread (local or distant), and the patient's medical history. Micropapillary carcinoma occurs when the initial tumour is smaller than $10 \mathrm{~mm}$ in diameter. The capacity to offer radioactive iodine treatment to ablate leftover thyroid, even metastatic disease, and the ability to follow up for tumour recurrence using serum thyroglobulin levels are all potential advantages of a total thyroidectomy. In cases when the tumour is less than $2 \mathrm{~cm}$ in diameter, unifocal, and does not extend beyond the thyroid gland, a hemi thyroidectomy is advised. As a surgical option for papillary thyroid cancer therapy, the research aims to compare hemi-thyroidectomy with whole thyroidectomy for recurrence rates, hypoparathyroidism and the frequency of re-operations due to recurrence. Methods: Papillary thyroid cancer was confirmed by FNAC and pathology reports prior to surgery in 40 patients at banha university hospital, 20 of them received hemi-thyroidectomy and the other 20 underwent complete thyroidectomy. From 2010 to 2020, the research will be conducted. Vocal cord palsy and tumour recurrence were shown to be indistinguishable between the two surgeries throughout the research period of up to three years. $(\mathrm{P}=1.00)$. Radioactive iodine ablation $(\mathrm{P}=0.487)$ is an option for management. Succeeding operations as an alternative method of care in the event that the disease returns $(\mathrm{P}=0.487)$ and recurrence-free survival. Patients who had a whole thyroidectomy were more likely to develop hypoparathyroidism than those who had a hemi-thyroidectomy. In spite of this, the P-value was just 0.02. A complete thyroidectomy for PTC treatment resulted in hypoparathyroidism in $30 \%$ of patients, either transitory or permanent. Hypoparathyroidism as a side effect of hemi-thyroidectomy has never been documented. For patients with papillary thyroid carcinoma who have been properly selected, hemi-thyroidectomy is an appropriate surgical option because it does not significantly differ from total thyroidectomy in terms of recurrent laryngeal nerve injury, the use of radioactive iodine, hypoparathyroidism and tumour recurrence rates.
\end{abstract}

Key words: Hemi, Thyroidectomy, Total Thyroidectomy, Papillary Thyroid Carcinoma

\section{Introduction}

More than $70 \%$ of all thyroid cancers are papillary thyroid carcinomas (PTCs), the most frequent kind of cancer [1].

Women are more likely than males to develop papillary carcinoma, with a female-to-male ratio of 2:1 and an average age of presentation between 30 and 40 years. [2] Lymphatic veins abound in the thyroid gland. In the peri- and pre-laryngeal nodes, the lymphatic veins drain first, and then proceed to the pre- and paratracheal nodes through the recurrent laryngeal nerve (RLN) and the mediastinal lymph nodes. [3] Environmental, genetic, and hormonal variables are all implicated in the development of PTC [1, 4]. Radiation has long been known to be a genotoxic component in PTC $[1,2]$.

The majority of people with hypothyroidism are asymptomatic and euthyroid, however a painless neck lump may develop over time [5]. Patients may arrive with lymph node metastases as their primary complaint [6]. Thyroid or lymph node FNAB confirms the diagnosis. To exclude out lymph node metastases in the central and lateral neck compartments, a full neck ultrasonography is highly indicated after a FNAB diagnosis of thyroid cancer. The presence of distant metastases at the time of the first presentation is infrequent. In terms of physical appearance, PTC may display a wide range of pathology. The majority of tumours are solid and gray-white in colour, while cystic tumours account for a significant portion of all cancers. Cystic lymph node metastases from a solid initial tumour is relatively rare. Tumor cells in the thyroid may either demonstrate a pattern of infiltrative growth or growth that extends directly to nearby tissues. Papillary structures with branching are seen in the traditional PTC $[1,2]$.

Cells with eosinophilic cytoplasm and swollen nuclei cover the papillae. Tumors may alter or completely alter the polarity of the cells inside them. In certain cases, there is evidence of squamous metaplasia. Thyroglobulin-rich lamellae are frequent in certain forms of PTC. This is not a universal feature of the disease. Conventional, Follicular variation, Tall cell, Columnar cell, Diffuse sclerosing, Solid, Clear cell, and Papillary microcarcinoma are all examples of PTC histopathological variants. [1,2].

Diagnosis of thyroid nodules is an important part of PTC management, as is determining the appropriate surgical therapy for each patient, as well as providing ongoing follow-up and adjuvant medications to enhance long-term results. The justification for a complete thyroidectomy in the treatment of PTC is obvious. 
Removal of the whole thyroid minimises the risk of leaving possible foci and tumour recurrence since PTC is often multifocal. A radioactive iodine treatment is also possible, allowing for the destruction of any remaining cancerous thyroid cells, as well as follow-up scans and measures of thyroglobulin. Patients with large, locally aggressive, multicentric, or metastatic papillary thyroid cancer need complete thyroidectomy, with excision of neighbouring implicated tissues, and appropriate nodal surgery, followed by radioiodine ablation with long-term TSH suppression. As a result, hemi-thyroidectomy should only be performed on individuals with tumours less than $2 \mathrm{~cm}$ in diameter (unifocal, absence of extrathyroidal spread, no evidence of nodal metastasis). There is a comparison to be made between hemi-thyroidectomy and whole thyroidectomy as a surgical strategy for the therapy of papillary thyroid cancer in terms: of recurrent laryngeal nerve damage and hypoparathyroidism.

\section{Patients and Methods}

\section{Type of study and study population:}

This is a retrospective study that had been including 40 patients diagnosed with papillary thyroid carcinoma proved by FNAC and pathology reports preoperatively who presented to benha university hospital during the period from 2010 to 2020. 20 patients underwent hemithyroidectomy (group A) and the other 20 patients underwent total thyroidectomy (group B).

\section{Methods:}

\section{Data collected included:}

\section{History}

- Personal data: Name, age, sex, occupation, address...

- Detailed Present history including history of swelling(s), symptoms of hypo or hyperthyroidism and compression symptoms

- History of medical diseases: Diabetes, Hypertension and bronchial asthma.

- Date of admission in the hospital.

- Past history (previous operations and regular drugs intake)

Clinical examination:

\section{General and local examination:}

- Vital signs, cardiovascular, neurological and respiration assessment.

- Examination of head and neck

\section{B-Investigations:}

1-laboratory:

- Complete blood count (CBC).

- Thyroid function tests: TSH, freeT3and T4

- Liver function tests (AST, ALT, ALP, serum bilirubin, serum albumin)

- Kidney functions tests (Serum urea and creatinine).
- Coagulation profile: PT, PTT, INR.

- Random blood sugar.

- Virology markers HBsAg, HCV Ab.

- HBA1C. for diabetic patients.

- Electrocardiography (ECG).

2-radiological:

- Neck ultrasound.

- CT scan.

- Echocardiography.

3-Biopsy: fine needle biopsy and its pathology report.

Surgical Procedures:

Anesthesia: general anesthesia.

Position: the patient is supine on the table on a head ring with a sand bag between the shoulders. Neck is extended.

Incision and exposure of the thyroid gland

A \#10 blade is used to pierce the skin when full sanitation and complete aseptic conditions have been met. The platysma is dissected through the subcutaneous fat using a scalpel and monopolar cautery. Upon determining the platysma's location, it is incised throughout the length of the incision. Using either sharp or blunt dissection, subplatysmal flaps are lifted up to the thyroid cartilage. When raising the subplatysmal flaps, care must be given not to injure the skin margins with the retractor or to lacerate or harm the anterior jugular vein (s)

Sternohyoid and sternothyroid strap muscles are discovered. Diathermy is then utilised to dissect into the deep cervical fascia (which covers the muscles) on both sides of the sternohyoid muscle, starting at its midline between the strap muscles. When big glands are invaded by the tumour, muscle cutting of the straps has been performed. To prevent damaging their neve supply, it is done at the highest feasible level. Muscles retract laterally to expose the gland if this is not possible. Thyroid tissue and the fascia that covers it may readily be seen in this dissection.

Once the thyroid gland has been identified, the focus shifts to a particular lobe of the thyroid. Strap muscles are pulled inwards to elongate the gland's neck, while the middle thyroid vein is ligated (if present).

Two ties are placed on the proximal stump of the superior thyroid artery with $2 / 0$ vicryl, dividing and tying the arteries under direct view. Avoiding injury to the external laryngeal nerve. Figure out what's going on here (1) Afterwards, the inferior thyroid veins are ligated. " The RLN and parathyroid glands may be seen from the posterolateral surface of the gland, which is turned medially. 
Using an operating microscope, the recurrent laryngeal nerve and the inferior thyroid artery are protected from harm during this procedure. One by one, the branches of the inferior thyroid artery are attached to the parathyroids.

The parathyroid glands may be identified.

It is normally attached to the top posterolateral side of the thyroid and is dissected using bipolar diathermy, conserving its own blood supply, to remove the upper parathyroid glands

A $1 \mathrm{~cm}$ radius surrounding the inferior pole of the thyroid gland, and nearly invariably anterior to the recurrent laryngeal nerve's plane, is the typical location of the inferior parathyroid glands. 3 to 7 percent of individuals may have additional glands, which is something to keep in mind. Strict care is used to remove each gland one by one, placing them on the thyroid bed.

The recurrent laryngeal nerve has been identified.

A few anatomic features may help identify nerves during surgery. The thyroid lobe's posterolateral side is marked by the Tubercle of Zuckerkandl, which is most typically present on the other side of the recurrent laryngeal nerve. More than nine-tenths of the recurrent laryngeal nerve's nerve endings are situated medial to the tubercle, which may be detected in $80 \%$ of thyroid tissues.

It is located in the tracheo-oesophageal groove and deep to the inferior thyroid artery branches, however it might be in front of or between its branches.

The thyroid gland is dissected, and a sufficient piece of the nerve is cut to assure its safety. Figure out what's going on here (2).

Once the recurrent laryngeal nerve is freed and can be viewed along its entire length, the remainder of Berry's ligament is divided by sharp dissection. (Berry's ligament defines the posterolateral attachment of the thyroid gland). Dissection across the front of the trachea, separating the gland and the isthmus using diathermy.

The lobe to be removed and the isthmus are now free. in total thyroidectomy, the procedure is repeated for the opposite side. Figure (3)

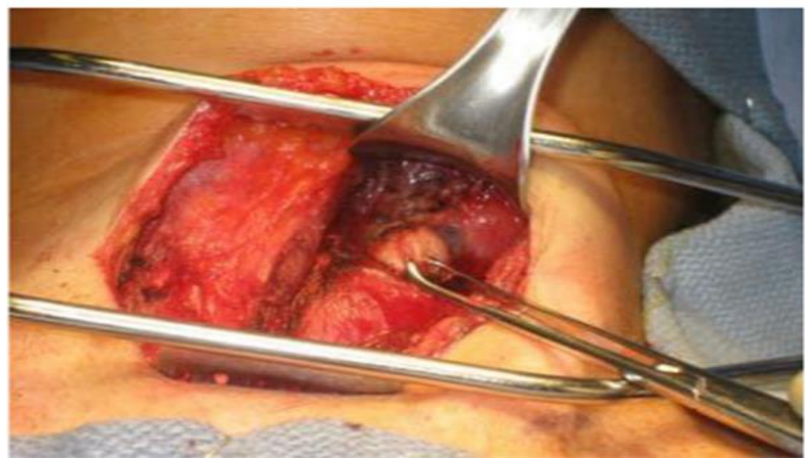

Fig. (1) Exposure of the superior pole.

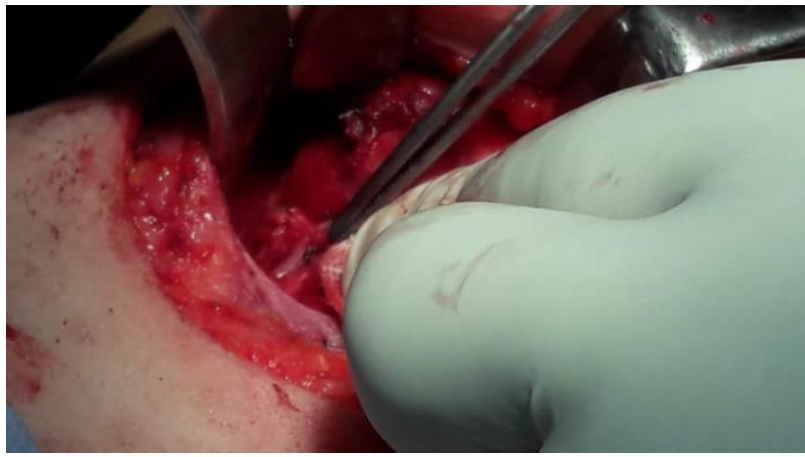

Fig. (2) Recurrent laryngeal nerve.

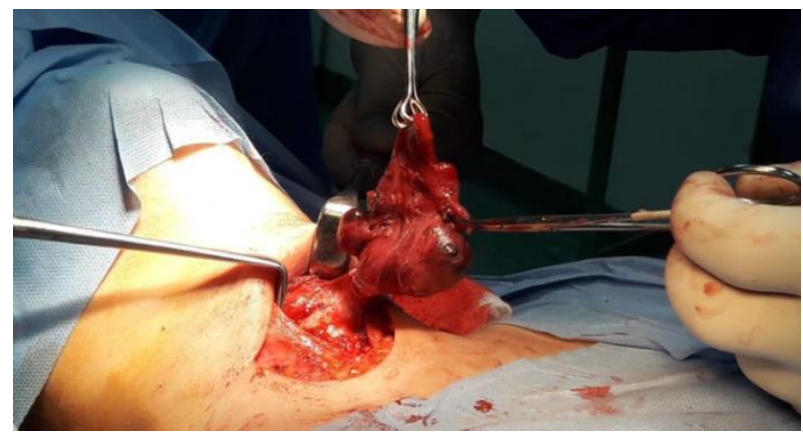

Fig. (3) Total thyroidectomy specimen 


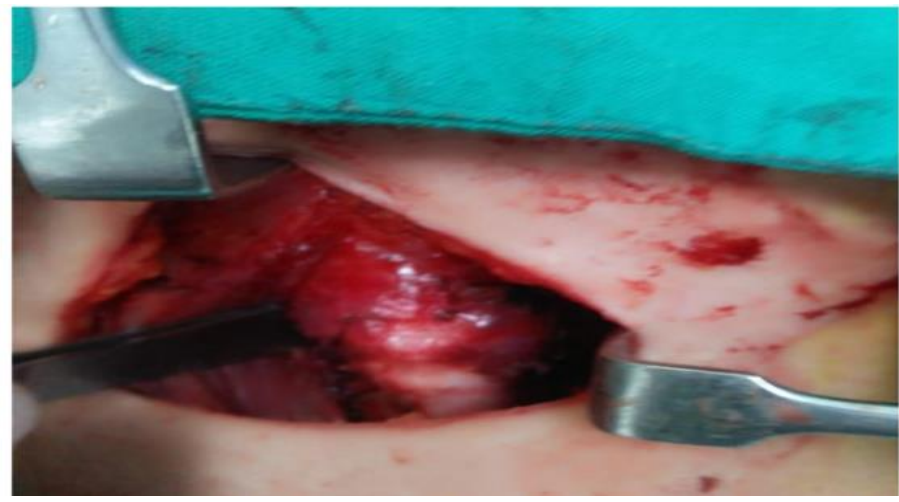

Fig. (4) After total thyroidectomy.

The tumour must be more than 2 centimetres in diameter, have lymph node involvement, and be multifocal in order to qualify for a complete thyroidectomy as a surgical option.

The tumour must be less than $2 \mathrm{~cm}$ in diameter, unifocal, not extend beyond the thyroid gland, and have no lymph node (s) dissemination to consider hemithyroidectomy as the surgical option.

An arterial forceps is inserted across the isthmus dividing the thyroid gland, and the specimen is extracted. For the stump, the running $2 / 0$ vicryl suture is employed. Histopathological tests are carried out on the material after it is removed.

Only in instances of complete thyroidectomy where there was evidence of lymphatic dissemination, lymph nodes were dissected (either clinically or by investigation or intraoperatively).

Level VI was dissected in four patients who had lymph nodes in the central neck compartment that tested positive.

Which has the following boundaries:

- superiorly: inferior border of hyoid bone

- Inferiorly: suprasternal notch.

- Laterally: medial borders of both common carotids

- posteriorly: trachea.

\section{Closure}

Obtaining hemostasis in the thyroid bed is imperative. This is performed by using bipolar diathermy especially, in the vicinity of the recurrent laryngeal nerve. Figure (4). A suction drain has been used in all cases. Approximation of the strap muscles with $3 / 0$ vicryl and their overlying fascia.

Platysma is closed as a single layer.

Skin is closed by subcuticular sutures with $4 / 0$ vicryl.

\section{Data collected:}

- Operative data including: Injury to recurrent laryngeal nerve and hypoparathyroidism.

- Recurrence rate of the tumor is registered.

Postoperative staging is used to determine prognosis, make decisions regarding postoperative adjunctive therapy and follow-up, and enable accurate communication between health-care providers.
AJCC/ The Union for International Cancer Control (UICC) staging is used for all patients.

Follow-up

- For long-term follow-up care, a physical examination and a serum thyroglobulin level every 6-12 months are obtained. (Every serum thyroglobulin measurement included a quantitative thyroglobulin antibody assessment). In patients who have undergone less than total thyroidectomy and in persons who have had a total thyroidectomy but no radioactive iodine ablation regular neck ultrasonography is performed every 6-12 months.

- Yearly clinical examination and thyroglobulin measurements is the primary means of following low-risk patients who have undergone remnant ablation, demonstrated negative cervical ultrasonographic results and shown undetectable TSH-stimulated thyroglobulin.

- Cervical ultrasonography was performed 6-12 months after surgery to assess the thyroid bed and the central and lateral cervical nodal compartments.

Postoperative TSH suppression has been shown to improve outcomes in thyroid cancer. thyroid hormone treatment with a target TSH level of $(0.1-0.5 \mathrm{mIU} / \mathrm{L})$ slightly below the normal level is considered adequate for low-risk well differentiated thyroid cancer (WDTC). Data management and Statistical Analysis

Data collected throughout history, clinical examination, laboratory investigations and outcome measures coded, entered and analyzed using Microsoft Excel software. Data were then imported into Statistical Package for the Social Sciences (SPSS version 20.0) (Statistical Package for the Social Sciences) software for analysis. correlation by Pearson's correlation or Spearman's. $\mathrm{P}$ value was set at $<0.01$ for significant results.

\section{Results}

There was no statistically significant difference between males and females concerning the procedure done for treating PTC $(\mathrm{P}=1.00)$. Table (1)

There was no statistically significant difference between either procedure regarding incidence of vocal cord palsy. $(\mathrm{P}=1.00)$ Table $(2)$ 
Incidence of hypoparathyroidism was higher among patients who underwent total thyroidectomy compared to others who underwent hemithyroidectomy. However, this was statistically insignificant $(\mathrm{P}=0.02)$ as shown in table (3). $30 \%$ of patients who underwent total thyroidectomy for treating PTC suffered from hypoparathyroidism either transient or permanent. On the other hand, no one recorded suffered from hypoparathyroidism as a complication to hemi-thyroidectomy.

There was no statistically significant difference between either procedure regarding incidence of tumor recurrence in the study period up to 3 years. $(\mathrm{P}=0.487)$ Table (4).

There was no statistically significant difference between either procedure regarding use of radioactive iodine ablation as a management option. $(\mathrm{P}=0.487)$ Table (5).

There was no statistically significant difference between either procedure regarding re-operation for recurrence among the study population. $(\mathrm{P}=0.487)$ Table (6).

Table (1) the sociodemographic characteristics of the studied sample $(n=40)$.

\begin{tabular}{lcc}
\hline Variable & $\mathbf{N}$ & \% \\
\hline Thyroidectomy & 20 & 50 \\
Total & 20 & 50 \\
Hemi & & \\
Gender & 9 & 22.5 \\
Male (4 hemi,5 total) & 31 & 77.5 \\
Female(16 hemi,15 total) & $40.3 \pm 13.98$ & \\
Age (years) & &
\end{tabular}

Table (2) the association between the procedure done for treating PTC and vocal cord palsy complication among the study population $(n=40)$.

\begin{tabular}{lccc}
\hline VC palsy & Total & Hemi & P value \\
\hline & thyroidectomy & thyroidectomy & \\
Transient & $1(5 \%)$ & $1(5 \%)$ & 1.00 \\
Permanent & $1(5 \%)$ & 0 & \\
No & $18(90 \%)$ & $19(95 \%)$ & \\
\hline
\end{tabular}

Table (3) the association between the procedure done for treating PTC and incidence of hypo-parathyroidism among the study population $(\mathrm{n}=40)$.

\begin{tabular}{lccc}
\hline Hypoparathyroidism & $\begin{array}{c}\text { Total } \\
\text { Thyroidectomy }\end{array}$ & $\begin{array}{c}\text { Hemi } \\
\text { Thyroidectomy }\end{array}$ & P value \\
\hline Transient & $4(20 \%)$ & 0 & 0.02 \\
Permanent & $2(10 \%)$ & 0 & \\
No & $14(70 \%)$ & $20(100 \%)$ & \\
\hline
\end{tabular}

Table (4) the association between the procedure done for treating PTC and incidence of tumor recurrence among the study population $(n=40)$.

\begin{tabular}{lccc}
\hline Recurrence & $\begin{array}{c}\text { Total } \\
\text { thyroidectomy }\end{array}$ & $\begin{array}{c}\text { Hemi } \\
\text { thyroidectomy }\end{array}$ & P value \\
\hline Yes & 0 & $2(10 \%)$ & 0.487 \\
No & $20(100 \%)$ & $18(90 \%)$ & \\
\hline
\end{tabular}

Table (5) the association between the procedure done for treating PTC and the use of radioactive iodine ablation as a management option among the study population $(n=40)$.

\begin{tabular}{lccc}
\hline $\begin{array}{l}\text { Radioactive } \\
\text { ablation }\end{array}$ & $\begin{array}{c}\text { Total } \\
\text { thyroidectomy }\end{array}$ & $\begin{array}{c}\text { Hemi } \\
\text { thyroidectomy }\end{array}$ & P value \\
\hline Yes & $2(10 \%)$ & 0 & 0.487 \\
No & $18(90 \%)$ & $20(100 \%)$ & \\
\hline
\end{tabular}


Table (6) the association between the procedure done for treating PTC and re-operation for recurrence among the study population $(n=40)$.

\begin{tabular}{lccc}
\hline Re-operation & $\begin{array}{c}\text { Total } \\
\text { thyroidectomy }\end{array}$ & $\begin{array}{c}\text { Hemi } \\
\text { thyroidectomy }\end{array}$ & P value \\
\hline Yes & 0 & $2(10 \%)$ & 0.487 \\
No & $20(100 \%)$ & $18(90 \%)$ & \\
\hline
\end{tabular}

Table (7) the association between the procedure done for treating PTC and recurrence free survival among the study population up to 3 years $(n=40)$.

\begin{tabular}{lccc}
\hline $\begin{array}{l}\text { Recurrence- Free } \\
\text { Survival up to 3 years }\end{array}$ & $\begin{array}{c}\text { Total } \\
\text { thyroidectomy }\end{array}$ & $\begin{array}{c}\text { Hemi } \\
\text { thyroidectomy }\end{array}$ & P value \\
\hline Yes & $20(100 \%)$ & $18(90 \%)$ & 0.487 \\
No & 0 & $2(10 \%)$ & \\
\hline
\end{tabular}

There was no statistically significant difference between either procedure regarding recurrence free survival in the study period up to 3 years. ( $\mathrm{P}=0.487)$ Table (7).

\section{Discussion}

Treatment for PTC did not show a statistically significant difference between men and women $(\mathrm{P}=1.00)$. Age ranged from 40.3 to 13.98 years in the study.

According to Hirsch et al., [10] the final analysis comprised 161 patients, 60 of whom had hemithyroidectomy, and 101 of whom underwent complete thyroidectomy; hence, our results were consistent with their findings. Characteristics of the patient as a whole. There were no significant variations in age or gender distribution or the length of follow-up between the two groups (107).

In addition, Ji et al. [11] showed that in their investigation, there were no differences in gender or age between the two groups (total and hemi-thyroidectomy).

According to the results of this research, there was no statistically significant difference in the incidence of vocal cord palsy between the two procedures. a single instance in the hemi thyroidectomy group (A) and two in the whole thyroidectomy group (B)

Recurrent laryngeal palsy was documented by Hirsch et al., [10] as a permanent consequence of surgery in two patients in each group, with no statistically significant difference between the two groups.

Recurrent laryngeal nerve paralysis patients were more likely to have complete thyroidectomy than lobectomy, as shown by Kuba et al. [12], who performed the study.

Hypoparathyroidism was more common in individuals who received whole thyroidectomy than those who underwent hemi-thyroidectomy, according to the present research. In spite of this, the P-value was just 0.02. Patients who received a complete thyroidectomy for the treatment of PTC and developed hypoparathyroidism, either transitory or permanent, were 30 percent of the patients (6 patients). Hypoparathyroidism as a side effect of hemithyroidectomy has never been documented.

Only complete thyroidectomy patients were shown to have permanent hypoparathyroidism in a research by Ji et al. [11].
A research published in 2013 by Jeon et al. [13], found that transitory hypocalcemia ( 0 percent vs. 8.6 percent) and paralysis of the vocal folds were substantially more common in Group II (total thyroidectomy) than in Group I. (hemi- thyroidectomy). One patient in Group II had lifelong hypocalcemia, but both groups had no patients with chronic vocal fold paralysis.

Three patients who had complete thyroidectomy and one patient who had hemi- thyroidectomy had hypoparathyroidism, according to the research of Hirsch and colleagues [10]. During the second operation, the patient developed hypoparathyroidism as a result of questionable cytological findings in the remaining thyroid lobe. There was no statistically significant difference between the groups in terms of long-term problems.

When it came to the incidence of tumour recurrence in the study period, there was no statistically significant difference between either method. The hemi thyroidectomy group had just two documented recurrences (group a)

For recurrence of illness and overall mortality, the hemi- and complete thyroidectomy groups did not vary significantly, as shown by the research by Geron et al, [14] (OM).

Recurrence of PTC following hemi-thyroidectomy was detected in 11.4 percent of the 123 individuals studied by Huang et al. [15]. Recurrence rates of $4.6 \%$ were observed by Li and colleagues (16) in patients who had partial thyroidectomy, which is similar to the $4.6 \%$ reported by $\mathrm{Li}$ and colleagues (16). Ten percent of lowrisk patients were able to have surgery completed, according to Gershinsky et al. After a hemi- and a complete thyroidectomy, Kwon et al. [18] detected recurrence in $3.8 \%$ and $1.6 \%$ of patients with matching microscopic PTC ( $\mathrm{p}>0.01)$.

Only three of 289 patients investigated by $\mathrm{Wu}$ et al. [19] had recurrent illness following hemithyroidectomy, and all of them attained NED (No evidence of disease) after surgery. There was no difference in recurrence rates for PTC tumours of 1 to 4 $\mathrm{cm}$ in diameter $(85 \% 2 \mathrm{~cm})$ whether they were treated 
with hemi- or complete thyroidectomy, according to Kim et al. [20]. (6.1 percent vs. 5.7 percent , respectively).

An important landmark research by Bilimoria et al. [21] has sparked considerable debate, revealing the statistically significant benefit of complete thyroidectomy vs hemi-thyroidectomy for survival and recurrence.

Using radioactive iodine ablation as a treatment option, this research found that there was no statistically significant difference between either methods.

However, Hirsch et al. [10] found that patients who had a whole thyroidectomy attended the endocrine clinic more often than those who had a hemithyroidectomy $(\mathrm{P}=0.016)$. There were also appointments to arrange radioactive iodine therapy for people who had had complete thyroidectomy.

Radiation ablation with radioactive iodine (RAI) was also used in 92.4 percent of the thyroidectomy patients studied by Ji and colleagues [11].

Patients who had a whole thyroidectomy had higher rates of the following factors: age $>45$ years $(87$ percent vs 63 percent, respectively; $\mathrm{p}=0.002)$, nodal dissection (94 percent; 75\%), nodal metastasis (60\%) and larger tumours $(64 \%)$ than those who had thyroidlobectomy $(\mathrm{p}=0.022)$.

As far as re-operation for recurrence is concerned, the present research found no statistically significant difference between either treatment $(\mathrm{P}=0.487)$. In terms of Recurrence-Free Survival, there was no statistical difference between the two procedures. $(\mathrm{P}=0.487)$ up to three years of follow-up

Van Gerwen et al. [23] found good long-term prognosis in PTMC patients after surgery, with high recurrence-free survival rates in both the TT and TL groups. Small papillary thyroid cancers are often treated surgically, thus this is crucial to know. There is a trend toward better long-term survival for patients who undergo thyroid hormone replacement therapy (TT) over those who undergo thyroid surgery (LT), and this could be of clinical significance when deciding whether to proceed with life-long thyroid hormone replacement therapy (TT) or a subsequent thyroidectomy in the event of disease recurrence in the opposite lobe following LT.

Analysis of univariate and multivariate data by Jeon and colleagues [13], found that RFS in individuals with unilateral, multifocal PTMC was linked with the kind of surgical therapy (lobectomy vs. complete thyroidectomy). It was shown that there was no correlation between the kind of surgery and RecurrenceFree Survival (RFS).

Ji et al., [11] found that recurrence-free survival curves did not vary between the two groups of patients. There were 96.5 and 97.9 percent RFS in the complete thyroidectomy and hemi-thyroidectomy groups, respectively $(p=0.899)$. Subgroup analysis of tumour size did not show a difference in RFS rates (98.0 percent vs. 97.5 percent in tumours under 10 millimetres and 83.3 percent vs. $100 \%$ in those between 11 and 20 millimetres)
The conventional therapy for Papillary micro carcinoma was thyroidectomy for years, but active surveillance (AS) has evolved as an alternate method that entails ongoing monitoring in order to identify a minority of patients who may clinically advance and would benefit from rescue surgery.. Patients with lowrisk papillary thyroid carcinomas (PTCs) who are wellselected for surgical intervention are not at greater risk of recurrence or persistent illness if surgery is delayed. For low-risk papillary PTCs, AS trials conducted in Japan by the original researchers, and afterwards in numerous places throughout the globe, have shown that this method is safe [24].

Psychosocial variables, especially in less developed nations where only around one-quarter of patients seem to embrace AS, play a significant role in the application of AS in clinical practise [25].

There were only 34 (25 percent) of the 136 patients suitable for active monitoring who accepted this method, and roughly $10 \%$ of those who accepted abandoned it because of anxiety. (Smulever \& Pitoia, (26) Only 17 percent of patients had tumour growth following a median 4.6-year follow-up without evidence of distant metastases. In the 10 patients who had surgery after four years of active monitoring, there was no sign of illness in the median follow-up period.

\section{Conclusion}

No statistically significant difference was found between hemi-thyroidectomy and total thyroid removal as a surgical option for the management of papillary thyroid carcinoma in terms of recurrent laryngeal nerve injury, the use of radioactive iodine, hypoparathyroidism and recurrence rate of the tumour when it came to the surgical treatment of papillary thyroid carcinoma.

\section{References}

[1] A.Khan, RV.Lloyd, V.Nose, New York: Springer, Endocrine pathology: differential diagnosis and molecular advances, 2nd ed ,pp.181-236,2010.

[2] RA.DeLellis, RV.Lloyd, C.Eng, Lyon: International agency for research on cancer Press; Pathology and genetics of tumors of endocrine organs. World health organization. Classification of Tumors. $4^{\text {th }}$ ed.vol.10,pp.8190,2004.

[3] Y.Ito, C.Tomoda, T.Uruno, et al., Clinical significance of metastasis to the central compartment from papillary microcarcinoma of the thyroid. World J Surg. 30:91-99,2006.

[4] Li.Kari Hemminki Xinjun, International Journal of Cancer Familial risk of cancer by site and histopathology.vol.103,pp.105-9,2003.

[5] Lal.Geeta, H.Orlo Clark, McGraw Hill New York Thyroid Parathyroid,and Adrenal In. Schwartz's principles of surgery 11th edition.vol.2,pp.1647-1650.2019. 
[6] S.Norman Williams, J.K.Christopher Bulstrode , P.Ronan O'Connell, CRC press New York Neoplasms of the thyroid. In Bailey \& Love's Short Practice of Surgery, 26th Edition,pp.763-769,2013.

[7] HS.Ahn, HJ.Kim, HG.Welch, Korea's thyroidcancer "epidemic-screening and overdiagnosis. N Engl J Med.vol.371(19),pp.1765-1767,2014.

[8] SL.Cushing, CD.Palme, JL.Freeman et al., Prognostic factors in well-differentiated thyroid carcinoma. Laryngoscope.vol.114(12),pp.21102115,2004.

[9] S.David Cooper, M.Gerard Doherty, R.Bryan Haugen, American Thyroid Association (ATA) Guidelines Taskforce on Thyroid Nodules and Differentiated Thyroid Cancer, Revised American Thyroid Association management guidelines for patients with thyroid nodules and differentiated thyroid cancer.vol. (11),pp.1167$1214,2009$.

[10] D.Hirsch, S.Levy, G.Tsvetov, et al., Total versus hemithyroidectomy for small unilateral papillary thyroid carcinoma. Oncology letters.vol.7(3),pp.849-853,2014.

[11] YB.Ji, CM.Song, D.Kim, et al., Efficacy of hemithyroidectomy in papillary thyroid carcinoma with minimal extrathyroidal extension. European Archives of Oto-RhinoLaryngology.vol.276(12),pp.3435-42,2019.

[12] S.Kuba, K.Yamanouchi, M.Matsumoto, et al., Total thyroidectomy versus thyroid lobectomy for papillary thyroid cancer: comparative analysis after propensity score matching: a multicenter study. International Journal of Surgery.vol.38,pp.143-8,2017.

[13] YW.Jeon, HG.Gwak, J.Schneider, et al., Long-term prognosis of unilateral and multifocal papillary thyroid microcarcinoma after unilateral lobectomy versus total thyroidectomy. Annals of surgical oncology.vol.26(9),pp.2952-8,2019.

[14] Y.Geron, M.Shteinshneider, S.Koren, et al., Long-term outcome after hemithyroidectomy for papillary thyroid cancer: a comparative study and review of the literature. Cancers.vol.11(1),pp.26,2019.

[15] H.Huang, S.Liu, Z.Xu, et al., Long-term outcome of thyroid lobectomy for unilateral multifocal papillary carcinoma. Medicine (Baltimore) .vol.96,pp.e7461,2017.

[16] X.Li, C.Zhao, J.Gao, et al., Hemithyroidectomy increases the risk of disease recurrence in patients with ipsilateral multifocal papillary thyroid carcinoma. Oncol Lett. 5(4):1412-1416,2013.
[17] M.Gershinsky, O.Barnett-Griness, S.Grozinsky-Glasberg, et al., Total versus hemithyroidectomy for microscopic papillary thyroid cancer. J. Endocrinol. Investig.vol.35,pp.464-468,2012.

[18] H.Kwon, MJ.Jeon, DE.Song, et al., A comparison of lobectomy and total thyroidectomy in patients with papillary thyroid microcarcinoma: a retrospective individual riskfactor-matched cohort study. Eur J Endocrinol.vol.176,pp.371-378,2017.

[19] Z.G.Wu, X.Q.Yan, R.S.Su, et al., How many contralateral carcinomas in patients with unilateral papillary thyroid microcarcinoma are preoperatively misdiagnosed as benign? World J. Surg.vol.41,pp.129-135,2017.

[20] SK.Kim, I.Park, JW.Woo, et al., Total thyroidectomy versus lobectomy in conventional papillary thyroid microcarcinoma: Analysis of 8,676 patients at a single institution. Surgery.vol.161,pp.485-92,2017.

[21] K.Y.Bilimoria, D.J.Bentrem, C.Y.Ko ,et al., "Extent of surgery affects survival for papillary thyroid cancer," Annals of Surgery.vol.246,pp.375-381,2007.

[22] S.Kuba, K.Yamanouchi, M.Matsumoto, et al Total thyroidectomy versus thyroid lobectomy for papillary thyroid cancer: comparative analysis after propensity score matching: a multicenter study. International Journal of Surgery. 2017 Feb 1.vol.38,pp.143-8,2017.

[23] M.van Gerwen, M.Alsen, C.Sinclair, et al. Recurrence-free survival after total thyroidectomy and lobectomy in patients with papillary thyroid microcarcinoma. Journal of Endocrinological Investigation.vol.44(4),pp.725$34,2021$.

[24] AR.Shaha, RM.Tuttle, Editorial: Risk of disease progression during active surveillance of papillary thyroid cancer. Surgery.vol.163,pp.5354,2018

[25] RM.Tuttle, L.Zhang, A.Shaha, A clinical framework to facilitate selection of patients with differentiated thyroid cancer for active surveillance or less aggressive initial surgical management. Expert Rev Endocrinol Metab.vol.13,pp.77-85,2018.

[26] A.Smulever, F.Pitoia, Active surveillance in papillary thyroid carcinoma: not easily accepted but possible in Latin America. Arch Endocrinol Metab.vol.63,pp.462-469,2019. 